\title{
Desenvolvimento e validação de um assistente virtual para farmacovigilância de vacinas
}

\author{
Andréia Roque de Souza Cavalcanti ${ }^{1,2}$, João Antônio Correa ${ }^{1}$, José William Araújo \\ do Nascimento ${ }^{2,3}$, Rafael Roque de Souza ${ }^{2,3}$, Geicianfran da Silva Lima Roque ${ }^{2,3}$, \\ Sérgio Ricardo de Melo Queiroz ${ }^{3}$
}

${ }^{1}$ Faculdade de Medicina do ABC - Santo André, SP - Brasil

${ }^{2}$ Imune Research Lab, João Pessoa, PB - Brasil

${ }^{3}$ Centro de Informática, Universidade Federal de Pernambuco (Cin-UFPE) - Recife, PE - Brasil

andreia@imunekids.com.br, cor.jantonio@gmail.com, \{jwan; rrs; gslr;
srmq\} dcin.ufpe.br

\begin{abstract}
This study was carried out to develop and validate a virtual assistant for the purpose of performing pharmacovigilance of vaccines. This is a quantitative methodological research conducted in three phases: 1) survey of the scientific literature; 2) content validation through a panel of 22 health professionals; 3) implementation of an automated messaging virtual assistant. As obtained results, the content domains of the virtual assistant achieved excellent adequacy, relevance, and representativeness criteria, all above $86 \%$; the content validity index obtained an average of 0.90 and an average Kappa value of 0.76 .
\end{abstract}

Resumo. Este estudo foi realizado para desenvolver e validar um assistente virtual com a finalidade de realizar a farmacovigilância de vacinas. Trata-se de uma pesquisa metodológica quantitativa realizada em três fases: 1) levantamento da literatura científica; 2) validação do conteúdo através de um painel de 22 profissionais de saúde; 3) implementação de um assistente virtual de mensagens automatizadas. Como resultados obtidos, os domínios do conteúdo do assistente virtual alcançaram critérios de adequação, relevância e representatividade excelentes, todos acima de 86\%; o indice de validade de conteúdo obteve uma média de 0,90 e um valor médio de Kappa de 0,76.

\section{Introdução}

A vacina é uma das maiores criações da medicina contemporânea e continua sendo a forma mais segura e eficaz de prevenção, principalmente contra as doenças infectocontagiosas. Embora as vacinas existentes sejam altamente eficazes, nenhuma delas está isenta de provocar reações nos indivíduos, isto porque, cada indivíduo reage a elas de modo diferente, seja pela condição de saúde apresentada, por diferentes antecedentes genéticos ou por causas ainda desconhecidas [Xie and Ele, 2017].

Estas reações são denominadas de eventos adversos pós-vacinais (EAPV), tratando-se de reações à saúde ou a um problema de saúde que ocorre durante ou após a administração de qualquer vacina. Estes eventos têm relação temporal e podem estar 
relacionados à própria vacina ou coincidentemente a elas, sendo que, neste último caso, não têm relação com a imunização [European Medicines Agency, 2020].

Nessa perspectiva, o objetivo da farmacovigilância é a detecção precoce de EAPV e a resposta adequada e oportuna a fim de minimizar os efeitos negativos à saúde dos indivíduos [SOLDATOU et al., 2019]. O uso de recursos tecnológicos móveis baseados em inteligência artificial pode incentivar a maior participação do paciente no processo de farmacovigilância. Estudos demonstraram que a participação ativa do paciente pode beneficiar os programas de vacinação, facilitar a notificação de EAPV e fornecer acesso a informações confiáveis sobre a vacina [Oosterhuis et al., 2018].

Uma tecnologia promissora neste sentido é o uso de assistentes virtuais ou agentes de conversação, programas que podem se comunicar com um ser humano usando linguagem natural por meio de comandos como a troca de mensagens por texto e voz [Ayanouz et al., 2020]. Diante das evidências sobre os benefícios dos assistentes virtuais aplicados à área de saúde [Milne-Ives et al., 2020] e da necessidade de identificar, acompanhar e orientar a população sobre as condutas diante de uma reação adversa pósvacinal, este estudo objetivou desenvolver e validar o conteúdo de um assistente inteligente para a farmacovigilância de vacinas.

\section{Métodos}

\subsection{Fases de desenvolvimento}

Trata-se de uma pesquisa metodológica realizada em três grandes etapas: 1) Revisão da literatura científica; 2) Definição e validação de conteúdo do assistente virtual; 3) Desenvolvimento do assistente virtual tipo assistente virtual para farmacovigilância de vacinas.

A primeira etapa foi caracterizada pelo levantamento da literatura científica para fundamentar as condutas relacionadas à temática proposta, considerando-se também a experiência clínica dos pesquisadores. Foram identificados os seguintes parâmetros para composição do assistente virtual: coleta de dados, classificação do caso conforme a gravidade (grave, não grave), intensidade (leve, moderado, intenso), previsibilidade (descrito ou não em bula), avaliação de causalidade (coincidente ou inclassificável) e avaliação de causalidade do EAPV (relação temporal entre a administração da vacina e o evento ocorrido). Para viabilizar a notificação das reações graves e esperadas das vacinas, foi utilizado como base o conteúdo presente no Manual de vigilância epidemiológica de eventos adversos pós-vacinação do Ministério da Saúde [Brasil, 2020].

Após a revisão do conteúdo voltada a farmacovigilância de vacinas, estes conteúdos foram definidos e passados por um processo de validação de conteúdo, através de um painel de especialistas formado por 22 profissionais de saúde (enfermeiros e médicos) experts em imunização, infectologia e virologia, com a finalidade de avaliar a relevância, adequação conceitual, pertinência, abrangência e clareza dos 5 domínios, 19 itens e duas figuras presentes no assistente virtual. Os domínios do assistente virtual se referem a seus eixos temáticos, descritos na tabela 1, enquanto os itens dos domínios são seus Requisitos Funcionais (RFs). Os RFs são as funções que o sistema deve fornecer, ou seja, como deverá reagir às entradas do usuário. 
Tabela 1: Domínios do assistente virtual

\begin{tabular}{cl}
\hline \multicolumn{1}{c}{$\begin{array}{c}\text { Domínios } \\
\text { (n de itens) }\end{array}$} & \multicolumn{1}{c}{ Descrição } \\
Domínio 1 (8 itens) & $\begin{array}{l}\text { Caracterização da reação adversa pós-vacinal } \\
\text { Caracterização da intensidade da reação adversa pós-vacinal } \\
\text { Domínio } 2 \text { (1 item) }\end{array}$ \\
Domínio 3 (3 itens) & $\begin{array}{l}\text { Caracterização da causalidade (coincidente) da reação adversa pós-vacinal } \\
\text { Orientações referentes às reações adversas pós-vacinais (cada item é } \\
\text { acionado pelo assistente virtual mediante as respostas identificadas nos } \\
\text { domínios anteriores) }\end{array}$ \\
Domínio 5 (1 item) & Satisfação final do usuário com o atendimento \\
\hline
\end{tabular}

Após ter seu conteúdo validado, o assistente virtual para farmacovigilância de vacinas foi desenvolvido na plataforma IBM Watson Conversation, dado a sua capacidade de processamento de linguagem natural, geração e avaliação de hipóteses e aprendizagem baseada na evidência [IBM, 2019]. Assim, os mecanismos de inteligência artificial utilizados no assistente virtual foram: aprendizagem de máquina, que usa os dados para detectar padrões e ajustar as ações da máquina; e análise dos dados, estruturados ou não-estruturados, que são coletados, armazenados e interpretados por sistemas especializados. Ainda nesta etapa de desenvolvimento do assistente virtual, foram elaborados os intents. Uma intent, ou intenção, é a finalidade em que o usuário deseja chegar ao ter enviado uma mensagem, por exemplo, se o usuário enviar "Bom dia" ao assistente virtual, este deve saber que a intenção do usuário ao ter enviado aquela mensagem, foi de "saudação", ou, "início de diálogo. Para a elaboração destes intents recorremos ao roteiro com o fluxo de diálogos desenvolvido para registrar as intenções do usuário no diálogo. Para alimentar os intents, foram adicionadas palavras-chaves e respostas pré-definidas que auxiliem o usuário na retirada de dúvidas sobre reações vacinais que possam ter ocorrido após o uso de vacinas. Os usuários podem interagir com o assistente virtual usando botões predefinidos ou digitando suas respostas. Quando a intenção do usuário não é identificada (por ter digitado uma resposta não reconhecida), o assistente diz que não entendeu e repõe a pergunta, de modo que isso possa ocorrer a qualquer momento durante a conversa. $O$ fluxo da conversa foi programado de modo que seja fácil de entender para que o assistente virtual possa ser usado por pessoas de qualquer nível educacional.

\subsection{Análise dos dados}

A concordância dos especialistas do conteúdo do assistente virtual foi verificada de forma quantitativa por meio do Índice de Validade de Conteúdo por item (I-IVC). Este índice indica a proporção de concordância sobre a relevância de cada item, considerando-se um resultado maior ou igual a 0,80 como valor mínimo adequado. Desse modo, considera-se o seguinte padrão de avaliação: I-IVC $\geq 0,80$ excelente, I-IVC entre 0,60 e 0,79 bom, e IIVC $\leq 0,59$ ruim, sendo o item eliminado [Polit and Beck, 2006]. Para avaliar a abrangência do conteúdo como um todo foi verificado o Índice de Validade de Conteúdo por nível de escala e média dos I-IVCs avaliados (S-IVC/Ave); O S-IVC/Ave deve ser igual ou maior que 0,90 como o padrão para este índice de congruência média [Polit and Beck, 2006]. 
Para avaliar a confiabilidade entre as avaliações foi calculado o Interrater Agreement (IRA), que oferece uma perspectiva única de confiabilidade, já que lida com a consistência das avaliações e a concordância com a similaridade dos níveis absolutos de classificação. Para interpretação deste índice foi utilizado o recomendado por O'Neill [2017] de que valores de 0-0,30 indicam falta de concordância, de 0,31-0,50 indicam concordância fraca, $0,51-0,70$ uma concordância moderada, $0,71-0,90$ uma concordância forte e 0,91-1.0 indicam uma concordância muito forte. Além disso, foi utilizado o teste Fleiss Kappa como complemento importante ao IVC, uma vez que ajusta a concordância casual. Foi utilizada a seguinte interpretação dos valores: $>0,74$ - excelente; 0,60 a 0,74 - bom; 0,40 a 0,59 - regular; <0,40 - insuficiente [Nichols et al., 2010]. Este estudo foi aprovado pelo Comitê de Ética em Pesquisa da Universidade Federal da Paraíba, (Número do Parecer: 4.426.034) e obedeceu aos preceitos do Código de Ética da Associação Médica Mundial (Declaração de Helsinque).

\section{Resultados}

\subsection{Validação de conteúdo}

Os 22 especialistas (enfermeiros: 15 - 68,2\%; médicos: 7 - 31,8\%) que participaram do painel de validação de conteúdo do assistente virtual de farmacovigilância de vacinas tinham uma média de idade de 42 anos (desvio padrão: 9,38), 86,3\% são do sexo feminino, 72,7\% possuem mais de 11 anos de experiência clínica na área do conteúdo analisado e são oriundos de quatro das cinco regiões brasileiras, sendo a região Nordeste a mais prevalente $(77,2 \%)$. Os domínios do conteúdo do assistente virtual alcançaram critérios de adequação, relevância e representatividade excelentes, todos acima de $86 \%$, com destaque para os domínios 1 e 4.

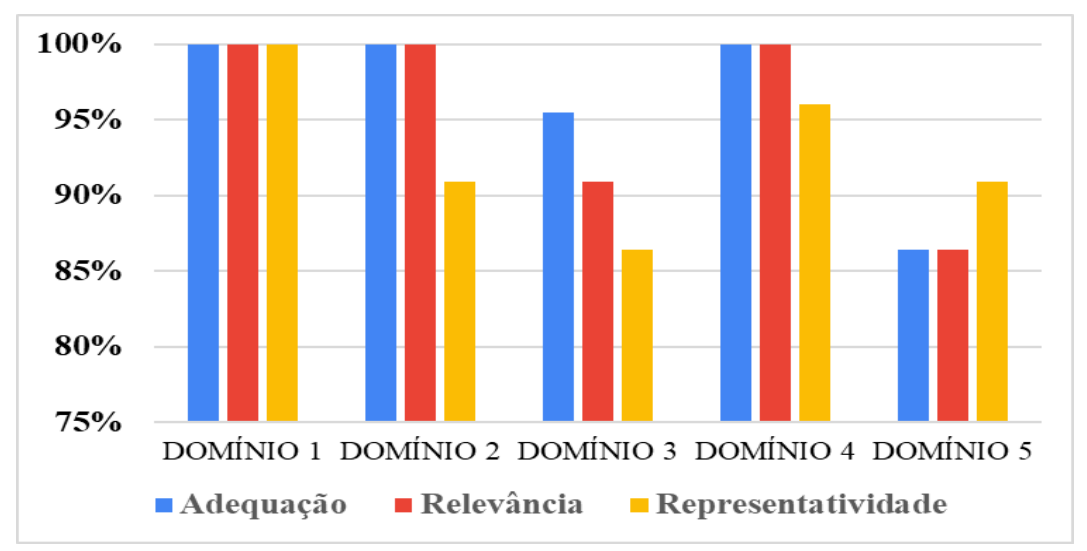

Figura 1: Avaliação dos critérios de adequação, relevância e representatividade das definições operacionais do script do assistente virtual para farmacovigilância de vacinas

Os 19 itens do assistente virtual tiveram valores de IVC acima de 0,80 (valor mínimo: 0,81; valor máximo: 0,98), indicando excelente relevância do conteúdo. A avaliação da validade de conteúdo como um todo, pelo método de S-IVC/Ave alcançou uma média de 0,90. A concordância inter-avaliadores foi muito forte (IRA: 1,00) à semelhança dos resultados do Fleiss Kappa, no qual se verifica que 17 dos 19 itens obtiveram concordância excelente, indicando concordância substancial entre os avaliadores. $O$ índice de Kappa médio foi de $0,76(p<0,05)$. 


\subsection{Protótipo de assistente virtual para farmacovigilância de vacinas}

Após a validação de conteúdo, o assistente virtual para farmacovigilância de vacinas foi desenvolvido na plataforma IBM Watson Conversation. Até o momento, o mesmo foi desenvolvido para ser utilizado na versão interface web, por meio de um navegador, podendo ser utilizado tanto em dispositivos móveis como em desktops. A interface, meio pela qual o usuário se comunica com o assistente virtual, foi criada buscando maximizar a interatividade e a experiência do usuário. $\mathrm{O}$ assistente é representado através de uma imagem feminina. $\mathrm{O}$ assistente virtual é operado por um fluxo de perguntas onde o usuário tem a possibilidade de informar o perfil do paciente que recebeu a vacina (idade), a forma de aplicação e o tipo de vacina que recebeu, bem como se apresentou algum tipo de reação e qual a sua intensidade (por meio de uma escala analógica). Além destes detalhes, o assistente virtual dialoga com o usuário a respeito de sintomas gripais prévios que podem interferir na interpretação da conduta final. Após analisar as respostas dos usuários, o assistente virtual fornece dicas e orientações acerca do que fazer diante da reação pósvacinal apresentada, bem como oferece a opção de o usuário entrar em contato com profissionais de saúde para maiores informações, se necessário.

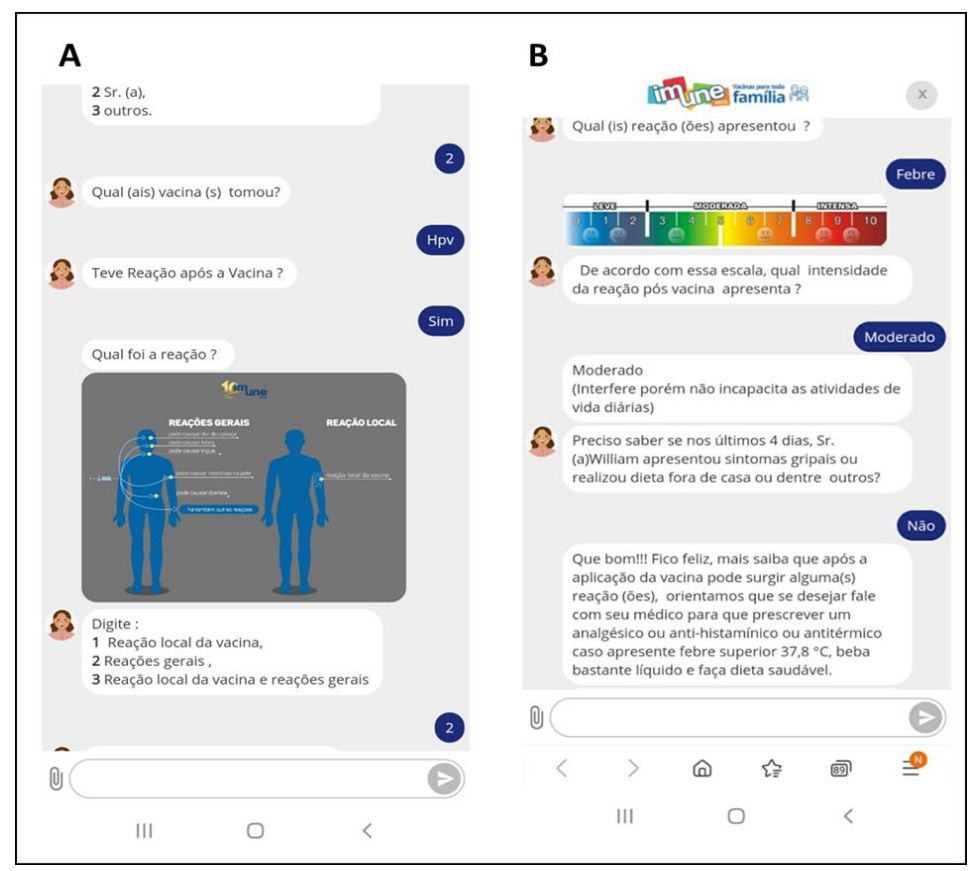

Figura 2: Interface web do assistente virtual: A: Avaliação do tipo de vacina que o usuário recebeu e qual a forma da reação; B: Avaliação da reação pós-vacinal e da sua intensidade, seguido das orientações para o caso.

\section{Considerações finais}

O assistente virtual para farmacovigilância de vacinas teve seu conteúdo validado, obtendo um nível satisfatório de validação, conferindo ao recurso tecnológico maior credibilidade e segurança para ser utilizado como um sistema de monitoramento ativo das reações adversas advindas das vacinas. Informações a respeito das reações adversas às vacinas contra COVID-19 utilizadas no Brasil estão sendo compiladas e incorporadas ao assistente virtual, fornecendo um guia de segurança para os indivíduos que receberam doses destas vacinas, sendo um recurso tecnológico que pode auxiliar no registro de informações e orientações sobre possíveis reações, deixando a população mais segura e 
confiante nos imunizantes contra o novo coronavírus. Salientamos que o protótipo desenvolvido já está pronto para os próximos testes, com usuários para verificações adequadas de confiabilidade e usabilidade.

\section{Referências}

Ayanouz, S., Abdelhakim, B.A. and Benhmed, M. (2020) A Smart Chatbot Architecture based NLP and Machine Learning for Health Care Assistance. Proceedings of the 3rd International Conference on Networking, Information Systems \& Security; 78, p. 1-6. Disponível em: https://doi.org/10.1145/3386723.3387897

Brasil. Ministério da Saúde. (2020) Manual de vigilância epidemiológica de eventos adversos pós-vacinação. Ministério da Saúde, Secretaria de Vigilância em Saúde, Departamento de Imunizações e Doenças Transmissíveis. 4. ed. Brasília: Ministério da Saúde. Disponível em: http://bvsms.saude.gov.br/bvs/publicacoes/manual_vigilancia_epidemiologica_event os_vacinacao_4ed.pdf

European Medicines Agency (2020) Guideline on good pharmacovigilance practices (GVP). Disponível em: https://www.ema.europa.eu/en/documents/regulatoryprocedural-guideline/guidelines-good-pharmacovigilance-practices-gvpintroductory-cover-note-last-updated-revision-3_en.pdf

IBM (2019) “IBM Watson”, Disponível em: https://www.ibm.com/Watson/APIs

Milne-Ives, M., Cock, C., Lim, E., Shehadeh, M.H., Pennington, N., Mole, G., et al. (2020) The Effectiveness of Artificial Intelligence Conversational Agents in Health Care: Systematic Review. J Med Internet Res; 22 (10), p. e20346, Disponível em: http://dx.doi.org/10.2196/20346

Nichols, T.R., Wisner, P.M., Cripe, G., et al. (2010) Putting the Kappa Statistic to Use, Qual Assur J 13, p. 57-61. Disponível em: https://doi.org/10.1002/qaj.48

O'Neill., T.A. (2017) An Overview of Interrater Agreement on Likert Scales for Researchers and Practitioners. Frontiers In Psychology, 8(1), p. 1-15. Disponível em: http://dx.doi.org/10.3389/fpsyg.2017.00777

Oosterhuis, I., Taavola, H., Tregunno, P.M., Mas, P., Gama, S., Newbould, V., et al. (2018) Characteristics, Quality and Contribution to Signal Detection of Spontaneous Reports of Adverse Drug Reactions Via the WEB-RADR Mobile Application: A Descriptive Cross-Sectional Study. Drug Saf. 41 (10), p. 969-978, Disponível em: http://dx.doi.org/10.1007/s40264-018-0679-6

Polit, D.F., and Beck, C.T. (2006) The content validity index: Are you sure you know what's being reported? critique and recommendations, Res Nurs Health; 29(5), p. 48997. Disponível em: https://doi.org/10.1002/nur.20147

Soldatou, V., Soldatos, A. and Soldatos, T. (2019) Examining Socioeconomic and Computational Aspects ofVaccine Pharmacovigilance. BioMed Research International; 1-15. Disponível em: http://dx.doi.org/10.1155/2019/6576483

Xie, J. and Ele, Y. (2017) Ontology-Based Vaccine Adverse Event Representation and Analysis. Adv Exp Med Biol; 1028, p. 89-103. Disponível em: http://dx.doi.org/0.1007/978-981-10-6041-0_6 